\title{
PLANE FLOWS WITH FEW STAGNATION POINTS ${ }^{1}$
}

\section{BY ANATOLE BECK}

Communicated by G. A. Hedlund, June 14, 1965

Introduction. This announcement deals with the subject of continuous flows in the Euclidean plane. It has long been known (cf. [1]) that every closed set in the plane is the invariant set of some continuous flow. In a recent paper [2], this author has shown that the same statement is not true for flows all of whose orbits are closed in the plane. In fact a necessary and sufficient condition is given for a closed set to be the invariant set of such a flow.

The author here announces new results in the same direction. The flows now dealt with are more general than those with closed orbits. They include flows with no stagnation points (see definition below), finitely many stagnation points, and countably many stagnation points. Results similar to those for flows with closed orbits are obtained for flows with no stagnation points or finitely many. On the other hand, every closed set can be exhibited as the invariant set of a flow with countably many stagnation points.

Definitions. A continuous flow in a topological space $X$ is a continuous mapping $\phi$ from $R \times X$ onto $X$ which satisfies the group property:

$$
\forall t_{1}, t_{2} \in R, \quad \forall x \in X, \quad \phi\left(t_{1}, \phi\left(t_{2}, x\right)\right)=\phi\left(t_{1}+t_{2}, x\right) .
$$

For each $t \in R$, the mapping $\phi_{(t)}$ defined by $\phi_{(t)}(x)=\phi(t, x)$ is a homeomorphism, and these homeomorphisms form a topological group which is a continuous homomorphic image of the real line.

For each $x \in X$, we define the set $\mathcal{O}(x)=\{\phi(t, x) \mid t \in R\}$, called the orbit of $x$. If $\mathcal{O}(x)=\{x\}$, then $x$ is called an invariant point of $\phi$, and we denote the set of invariant points of $\phi$ as $F(\phi)$, the invariant set of $\phi$. For each $x \in X, \mathcal{O}(x)$ is either a single point, a simple closed curve, or a 1-1 continuous image of the real line. We call these last two a circle and a line respectively, using the terms genuine circle and straight line when we mean these. Suppose $\theta(x)$ is a line, and we can find a point $y \in X$ and a sequence $\left\{t_{n}\right\}$ converging to $+\infty$ (resp. $-\infty$ ) such that $\phi\left(t_{n}, x\right) \rightarrow y$. Then $y$ is called an endpoint of $\mathcal{O}(x)$ at the positive (resp. negative) end. If $y=\lim _{t \rightarrow+\infty} \phi(t, x)$, then $y$ is a strong endpoint (at the positive end), with a similar definition at the negative end.

1 The research of this paper was supported by the Wisconsin Alumni Research Foundation and by the National Science Foundation. 
If not, then $y$ is a weak endpoint (at the appropriate end). If, in addition to being an endpoint of $\Theta(x), y \in F(\phi)$, then $y$ is a stagnation point of $\mathcal{O}(x)$, and also of $\phi$. If $y$ is a weak endpoint of $\mathcal{O}(x)$ at either end, then $y$ is a complex stagnation point of $\mathcal{O}(x)$, and also of $\phi$. If $y$ is a stagnation point of $\theta(x)$ (resp. of $\phi$ ), and $y$ is not a complex stagnation point of $\Theta(x)$ (resp. of $\phi$ ), then $y$ is a simple stagnation point of $\theta(x)$ (resp. of $\phi$ ).

The results here announced deal with the case where $X=E^{2}$, the Euclidean plane. If $C \subset E^{2}$ is open and simply connected, we call it a disc. If $C$ is open and multiply connected, but finitely connected, we call it a multiple annulus, and if it is doubly connected, we call it a simple annulus, or merely an annulus.

If $A$ and $C$ are any sets in $E^{2}$, and if there is a line lying in $C$ having a strong endpoint in $A$, then we say that $C$ is arcwise connected to $A$, and that the endpoint is arcwise accessible in $C$. If $F \subset C$, and $C$ is arcwise connected to $A$, while $C-F$ is not, then we say that $F$ shields $A$ in $C$.

Let $C$ be a disc or multiple annulus, and let $F \subset C$ be closed in $C$. If every component of $F$ is compact and every component of $C-F$ is an annulus, then $F$ is called a $T$-set of $C$. Let $U_{i} C_{i} \subset C$ be a disjoint union of countably many sets (allowing also none or finitely many), of which finitely many (possibly none) are multiple annuli and the rest are discs. Assume that $\bar{U} \cap \partial(C) \neq \square$ for every component $U$ of $C-U_{i} C_{i}$. For each $i$, let $F_{i}$ be a $T$-set of $C_{i}$. If $F=U_{i} F_{i}$ is closed in $C$, then we say that $F$ is a $T_{\sigma}$-set of $C$, and $U_{i} C_{i}$ is a setting for the $T_{\sigma}$-set $F$ in $C$.

Theorem A. Let $F$ be a closed set in $E^{2}$. Then $F$ is the invariant set of a flow with no stagnation points at all (not even at $\infty$ ) iff $F$ is the invariant set of a flow with compact orbits iff $F$ consists of its unbounded part $F_{\infty}$ together with a $T$-set in each component disc of $E^{2}-F_{\infty}$.

THEOREM B. $F$ is the invariant set of a flow with no stagnation points except possibly $\infty$ iff $F$ is the invariant set of a flow with closed orbits iff $F$ consists of its unbounded part $F_{\infty}$ and in each component disc $C$ of $E^{2}-F_{\infty}, F \cap C$ is a $T$-set or a $T_{\sigma}$-set which does not shield $\infty$ if $C$ is arcwise connected to $\infty$, while $F \cap C$ is a $T$-set of $C$ if $C$ is not arcwise connected to $\infty$.

Theorems A and B are proved by a sharpening of the techniques and methods used in [2]. The rather clumsy alternative in the statement of Theorem B can be eliminated by the concept of a regular setting for a $T_{\sigma}$-set in a disc or multiple annulus. 
Definition. Let $C$ be a disc or a multiple annulus, and let $F$ be a $T_{\sigma}$-set of $C$. Let $U_{i} C_{i}$ be a setting for $F$ in $C$, and let $S$ be any set disjoint from $C$. Assume that

$1^{\circ} \bar{C}_{i} \cap \bar{C}_{j}=\square, \forall i \neq j$,

$2^{\circ}$ for every component $D$ of $\partial(C)$ and each $C_{i}, \bar{C}_{i} \cap D=\square$ or $D$ is a component of $\partial\left(C_{i}\right)$,

$3^{\circ}$ if $\bar{C}_{i} \cap D=\square, \forall i$, then $\exists x \in S \cap D$ with $x$ arcwise accessible in $C-U_{i} \bar{C}_{i}$.

Then we call $U_{i} C_{i}$ a regular setting for $F$ in $C$ with respect to $S$, and $F$ is set regularly in $C$ with respect to $S$.

It is not hard to prove the following three lemmas:

Lemma. If $F$ is a $T$-set of $C$, then $F$ is a $T_{\sigma}$-set which is set regularly in $C$ with respect to any set $S$ disjoint from $C$.

LEMMA. If $C$ is a disc which is arcwise connected to $\infty$ and $F$ is a $T_{\sigma}$-set of $C$ which does not shield $\infty$, then $F$ is set regularly in $C$ with respect to $\{\infty\}$.

LEMMA. If $F$ is a $T_{\sigma}$-set which is set regularly in $C$ with respect to a set $S$ disjoint from $C$, and if $S$ is not arcwise accessible in $C$ or $F$ shields $S$ in $C$, then $F$ is a $T$-set of $C$.

We can now restate Theorem $B$ as

THEOREM $\mathrm{B}^{\prime} . A$ set $F$ is the invariant set of a flow with no stagnation points except possibly $\infty$ iff $F$ is the invariant set of a flow with closed orbits iff for every component disc $C$ of $E^{2}-F_{\infty}$, where $F_{\infty}$ is the unbounded part of $F, F \cap C$ is a $T_{\sigma}$-set of $C$ which is set regularly in $C$ with respect to $\{\infty\}$.

It is in this form that Theorem $B$ generalizes to

THEOREM C. Let $F$ be a closed set in $E^{2}$ and let $S \subset F$ be a finite set. Let $F_{0}$ be the union of the components of $F$ which intersect $S$. Then $E^{2}-F_{0}=U_{i} C_{i}$ is a disjoint union of finitely many multiple annuli and countably many discs. For each $C_{i}$, assume $F_{i}=F \cap C_{i}$ is a $T_{\sigma}$-set of $C_{i}$ which is set regularly in $C_{i}$ with respect to $S$. We see that $F=F_{0} \cup U_{i} F_{i}$. Then there is a flow $\phi$ such that

$1^{\circ} F$ is the invariant set of $\phi$,

$2^{\circ}$ every stagnation point of $\phi$ is an element of $S$,

$3^{\circ}$ every stagnation point of $\phi$ is simple.

Conversely, if $\phi$ is any flow with finitely many stagnation points, all of them simple, then the invariant set $F$ of $\phi$ is of the type described above, where $S$ is the set of stagnation points of $\phi$. 
Proof. We sketch a proof of Theorem $C$. The harder part is the construction. To accomplish it, we use the hypothesis of the regularity of the setting of $F$ in each $C_{i}$ to decompose $C_{i}$ into a disjoint union of discs and annuli. We do this by means of arcs which do not intersect each other or the sets of the setting, and which have strong endpoints in $S$. Then we define a concept of canonical slowness (the definition is too involved to take up here), and define on each of the arcs a flow which has only the endpoints for invariant points and which conforms to the standards of slowness set forth. We prove a sequence of interpolation theorems which allow us to extend the flows from the arcs into the discs and annuli they cut off in such a way that no new invariant points or stagnation points are created, all the stagnation points remain simple, and the interpolated flows conform to the same canons of slowness. The conjunction of all the flows thus interpolated will yield a flow in $C_{i}$ meeting the requirements of the theorem. The application of uniform standards of slowness to each disc and annulus in $C_{i}$ and uniformly for all the $C_{i}$ assures that the resulting flow is, in fact, continuous.

In the other direction, the theorem is based on the observation that the study of flows in the plane could equally well be the study of flows in the extended plane, with $\infty$ as an invariant point. If the extended plane is then considered as a sphere, we see that $\infty$ is deprived of its special properties, so that Theorem $\mathrm{B}^{\prime}$ can be considered as a theorem on flows in the sphere having at most one stagnation point. It is not too difficult to see that the same techniques, suitably modified, will yield some sort of characterization in the case of flows with finitely many stagnation points, especially if we have at our disposal the additional hypothesis that all the stagnation points are simple. The characterization turns out to be the one given.

A little further tinkering with the theorem, using the same methods, eliminates the need for considering the simplicity of the stagnation points.

THEOREM D. $A$ closed set $F$ is the invariant set of a flow with finitely many stagnation points iff it is the invariant set of a flow with finitely many stagnation points, all of them simple.

If we allow countably many stagnation points, then in general any closed set will serve for an invariant set, as we see in the next theorem.

THEOREM E. For any closed set $F \subset E^{2}$, we can find a flow $\phi$ such that

$1^{\circ} F$ is the invariant set of $\phi$,

$2^{\circ} \phi$ has only countably many stagnation points, 


\section{$3^{\circ}$ every stagnation point of $\phi$ is simple. ${ }^{2}$}

Thus, in the study of classes of flows in the plane, it seems that there are two large classes of hypotheses: those which produce a characterization for the invariant set based on $T$-set, $T_{\sigma}$-set, etc. and those which allow all closed sets as invariant sets. As we have shown in the cases studied in [2], such additional assumptions as requiring that the orbits be polygonal, or differentiable, or analytic do not change the characterizations. From the above, one would be tempted to assume that the significant difference is between those flows having finitely many stagnation points and those having infinitely many. This is not the case, however. Actually, one may naturally divide countable sets into those whose closures are also countable and those whose closures are uncountable. This distinction turns out to be significant in this study. For flows whose stagnation points form a set with countable closure, we can prove a theorem very similar to Theorem $\mathrm{C}$. This new theorem involves redefinition of all the terms used here, and we will not state it explicitly.

\section{REFERENCES}

1. Anatole Beck, On invariant sets, Ann. of Math. 67 (1958), 99-103.

2. - Plane flows with closed orbits, Trans. Amer. Math. Soc. 114 (1965), 539-551.

The Hebrew University of Jerusalem, Jerusalem, Israel and THE UNIVERSITY OF WISCONSIN

2 The author wishes to thank Mr. Jerome Dancis of the University of Wisconsin for supplying a portion of the proof of this theorem. 